\title{
Cycloaddition of Strained Cyclic Alkenes and Ortho-Quinones: A Distortion/Interaction Analysis
}

\author{
Jorge Escorihuela, Wilhelmus J. E. Looijen, Xiao Wang, Adelia J. A. Aquino, Hans Lischka,* \\ and Han Zuilhof*
}

Cite This: J. Org. Chem. 2020, 85, 13557-13566

Read Online

\section{ACCESS \\ 山ll Metrics \& More \\ 回星 Article Recommendations \\ Supporting Information}

ABSTRACT: The chemistry of strained unsaturated cyclic compounds has experienced remarkable growth in recent years via the development of metal-free click reactions. Among these reactions, the cycloaddition of cyclopropenes and their analogues to orthoquinones has been established as a highly promising click reaction. The present work investigates the mechanism involved in the cycloaddition of strained dienes to orthoquinones and structural factors that would influence this reaction. For this purpose, we use B97D density functional theory calculations throughout, and for relevant cases, we use spin component-scaled MP2 calculations and single-point domain-based local pair natural orbital coupled cluster (DLPNO-CCSD $(\mathrm{T})$ ) calculations. The outcomes are analyzed in detail using the distortion/interaction model, and suggestions for future experimental work are made.

\section{INTRODUCTION}

Since the coining of click chemistry by Sharpless and coworkers in 2001, ${ }^{1}$ the quest for novel reactions that fulfill the click requirements has experienced an exponential blossoming growth. This emergence of click chemistry has had a profound influence on almost all branches of chemical science, such as carbohydrate chemistry, ${ }^{2,3}$ polymer chemistry, ${ }^{4,5}$ radiochemistry, ${ }^{6,7}$ etc. From all of the reactions cataloged into the click chemistry library, copper(I)-catalyzed 1,3-dipolar azide-alkyne cycloaddition (CuAAC) emerged as the prototype click chemistry reaction as a consequence of its robustness, quantitative yield, and high chemoselectivity. ${ }^{8,9}$ This $\mathrm{Cu}$ catalyzed reaction has been widely used as an efficient functionalization strategy of polymeric materials and biomolecules for in vitro applications; ${ }^{10,11}$ however, the negative effects of the $\mathrm{Cu}$ catalyst have greatly limited its use with living systems. ${ }^{12}$ This has driven the development of metal-free, often strain-promoted reactions ${ }^{13}$ and photochemical $^{14}$ click reactions. Accordingly, light-activated thiol-ene and thiol reactions, ${ }^{15,16}$ Diels-Alder and a manifold of 1,3-dipolar cycloadditions, carbonyl condensations with oximes, ${ }^{17}$ thiol-Michael additions, ${ }^{18}$ and sulfur(VI) fluoride exchange $(\mathrm{SuFEx})^{19-22}$ reactions have been developed to overcome the use of metal catalysts. Among these novel metal-free reactions, strainpromoted cycloadditions have emerged as promising reactions due to their fast kinetics and good to excellent yields. These reactions include the strain-promoted azide-alkyne cycloaddition (SPAAC) reaction, ${ }^{23-25}$ the inverse electron-demand Diels-Alder (IEDDA) ${ }^{26-29}$ reactions such as the tetrazinetrans-cyclooctene (TCO)/cyclopropene click, ${ }^{30}$ strain-promoted alkyne-nitrone cycloaddition (SPANC), ${ }^{31,32}$ and the strain-promoted oxidation-controlled cyclooctyne-1,2-quinone cycloaddition (SPOCQ). ${ }^{33-37}$

While all of these reactions have significant potential in both biological and materials sciences, the size (and sometimes hydrophobicity) of the groups involved may hamper their effectiveness, especially in crowded environments. Therefore, smaller and bio-orthogonal probes-often termed mini-tagsare sought after. Among such small-sized targets, cyclopropenes are picking up considerable attention. Cyclopropenes have been shown to participate in fast bio-orthogonal ligation reactions with 1,2,3,4-tetrazines and photoclickable tetrazoles. ${ }^{38}$ In addition, our group has recently introduced the cycloaddition of 1-methyl-3-substituted cyclopropenes with a 1,2-quinone that displays similar reaction rates. ${ }^{39}$ Furthermore, the reaction displays quantitative conversion of the cyclopropenes toward cycloaddition products under mild conditions, suggesting its potential. This novel click reaction was efficiently applied for monolayer functionalization with quantitative yields for polymer brush modification. In this regard, the small size of the cyclopropene moiety turned out to be crucial in these crowded environments; with the larger cyclooctyne moiety, the yields were almost a factor of 2 lower. ${ }^{39}$ This situation is also of significant importance in

Received: July 14, 2020

Published: October 26, 2020 
Scheme 1. Reactions under Current Study Together with Relevant Cyclic Alkenes and o-Quinones, and a Schematic Depiction of the Concepts of Distortion and Interaction Energies

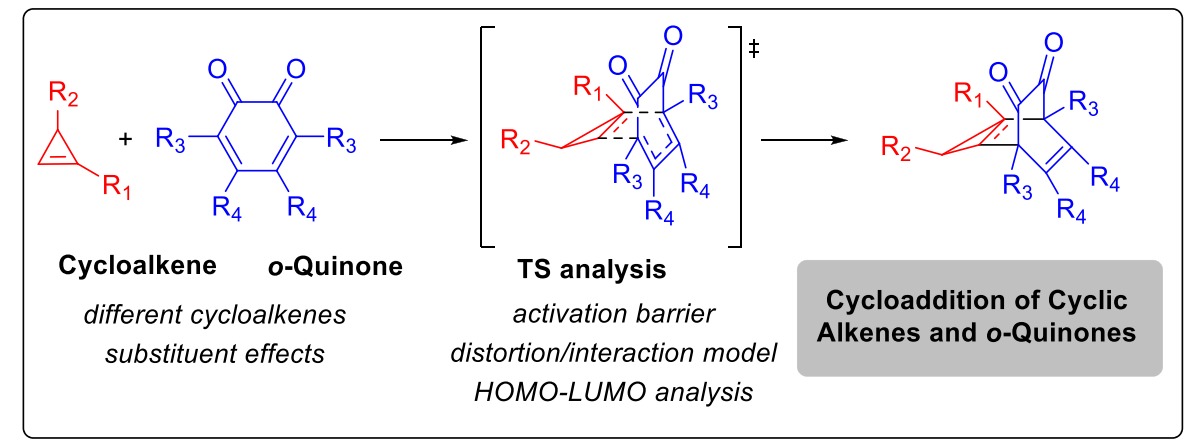

Distortion/interaction model
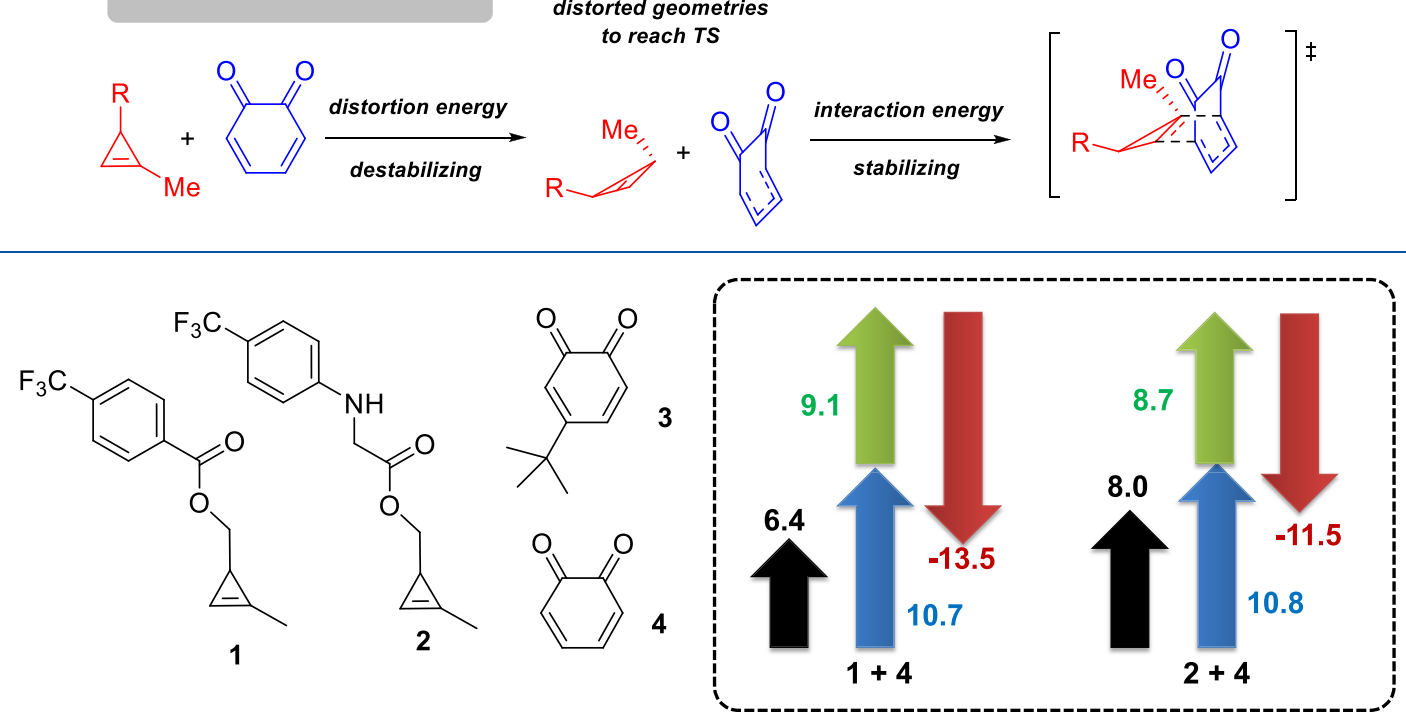

Figure 1. Chemical structures of compounds 1-4 and distortion/interaction analysis for $\mathbf{1}+\mathbf{4}$ and $\mathbf{2}+\mathbf{4}$ cycloadditions. Cyclopropene distortion energy (blue arrow), $o$-quinone distortion energy (green arrow), activation energy (black arrow), and interaction energy (red arrow). Calculated energies are shown in $\mathrm{kcal} / \mathrm{mol}$.

bioconjugation reactions, where small reagents are highly required. $^{40}$

Theoretical studies of such reactions are rapidly advancing due to a combination of two factors: First, the accuracy of such approaches continues to increase and can really complement experiment. ${ }^{41,42}$ Second, novel analysis methods help experimentally oriented organic chemists to better understand reaction mechanisms and chemical reactivity in terms of wellknown structural concepts. Specifically, the distortion/ interaction model $^{43,44}$ analyzes the barrier toward the transition state (TS) in terms of the (unfavorable) structural distortion of the reactants to reach that state and the (favorable) interaction that takes place between the reaction partners.

In the current study, we investigate the cycloaddition of a range of cyclopropenes and other cyclic dienes to orthoquinones by quantum chemical calculations (see Scheme 1). For this purpose, we use (based on their success in previous comparative theoretical studies) ${ }^{36}$ dispersion-corrected B97D density functional theory calculations, complemented by spin component-scaled (SCS)-MP2 calculations and a multiconfiguration method, namely, single-point domain-based local pair natural orbital coupled cluster (DLPNO)-CCSD(T) calculations. ${ }^{36}$ Finally, the distortion/interaction model is used to describe the influence of the reactant strain and approach toward the TS, so as to facilitate the further study and development of this recent click reaction.

\section{RESULTS AND DISCUSSION}

Reactivity of Different Cycloalkenes with o-Quinone. Starting from previously reported experimental results, which showed that a 1-methylcyclopropene derivative bearing the fluorinated ester 1 and carbamate $\mathbf{2}$ showed rapid kinetics $\left(k_{2}\right.$ $=1.95$ and $1.70 \mathrm{M}^{-1} \mathrm{~s}^{-1}$ for 1 and 2 , respectively) in the strainpromoted cycloaddition with $o$-quinones, ${ }^{39}$ we performed transition-state calculations at the B97D/6-311+G(d,p) level of theory. As shown in a previous work, the replacement of the experimentally used 4-tert-butyl-1,2-benzoquinone 3 by 1,2benzoquinone 4 did not have a significant effect on the reaction pathway of the cycloaddition. ${ }^{36}$ Consequently, 1,2benzoquinone 4 was used to simplify the calculations. Starting with compound 1 given its superior kinetics in solution, the cycloaddition was studied using this 1-methylcyclopropene as a model compound. The computed activation Gibbs energies for the endo-cycloaddition toward quinone 4 were lower $(\sim 1.5$ $\mathrm{kcal} / \mathrm{mol}$ ) than those of the exo-approach. Consequently, we focused only on the endo-cycloaddition for the compounds shown in this study. The calculations confirmed our 


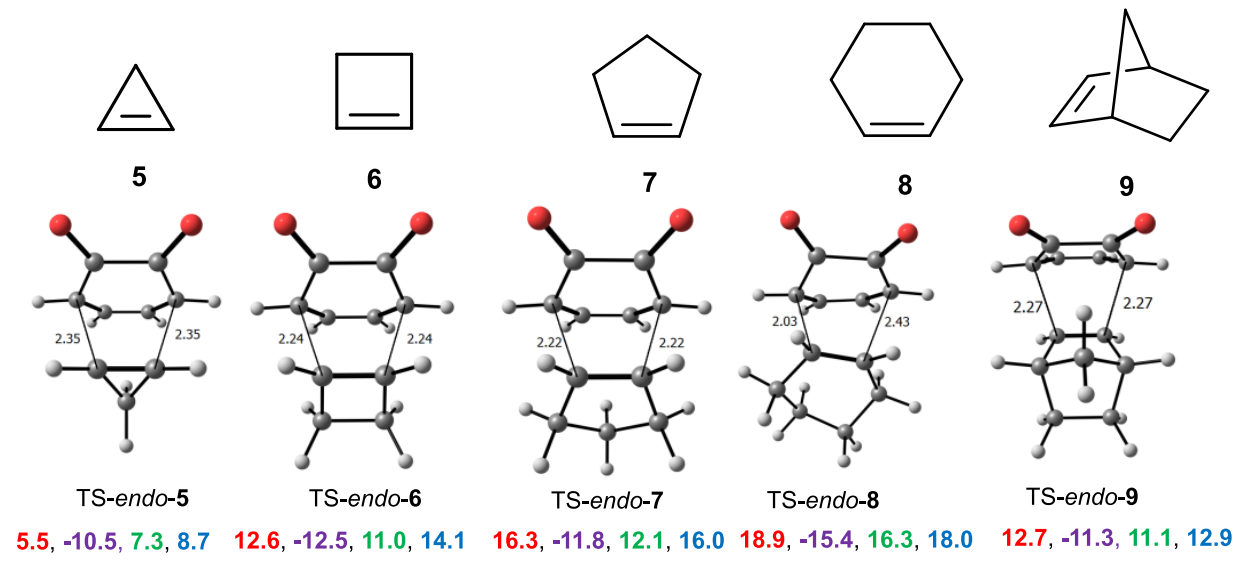

Figure 2. Activation energies (blue), interaction energies (purple), cyclopropene distortion energy (red), and $o$-quinone distortion energy (green) computed by means of $\mathrm{B} 97 \mathrm{D} / 6-311+\mathrm{G}(\mathrm{d}, \mathrm{p})$. All energies are given in $\mathrm{kcal} / \mathrm{mol}$ and distances in $\AA$.

observations $^{39}$ that the cycloaddition of $\mathbf{1}$ and $\mathbf{4}$ is always preferred on the face away from the 3 -substituent of the cyclopropene, given the asynchronous $\mathrm{C} \cdots \mathrm{C}$ distances for the formed $\mathrm{C}-\mathrm{C}$ bonds $\left(r(\mathrm{C} \cdots \mathrm{C})^{\mathrm{TS}}=2.12\right.$ and $\left.2.67 \AA\right)$. These findings showed that the activation barrier for the cycloaddition of 1-methylcyclopropene $\mathbf{1}$ with $o$-quinone via TS-1 is $6.4 \mathrm{kcal} / \mathrm{mol}$, which is $1.6 \mathrm{kcal} / \mathrm{mol}$ lower than that of 1 methylcyclopropene 2 via TS-2 (6.4 vs $8.0 \mathrm{kcal} / \mathrm{mol}$, Figure 1$)$. Both cycloadditions proceeded in an exergonic way with a Gibbs energy of reaction of around $-27 \mathrm{kcal} / \mathrm{mol}$. To gain more insights into the origins of the activation barriers of these reactions, we used the distortion/interaction model. ${ }^{43}$ As shown in Figure 1, the cyclopropene distortion energies are nearly identical, and only small differences arise when comparing both quinone distortion energies. Therefore, the differences in reactivity arise from differences in interaction energies, which are related to the degree of charge transfer in this cycloaddition reaction from the highest occupied molecular orbital (HOMO) of cyclopropene to the lowest unoccupied molecular orbital (LUMO) of o-quinone. Calculations show that the carbamate substituent in $\mathbf{2}$ decreased the HOMO energy with respect to cyclopropene 1 ( -9.96 vs $-9.78 \mathrm{eV}$ ), thereby increasing the HOMO-LUMO energy gap and concomitantly the transition-state energy, in line with experiment. With the aim to evaluate the accuracy of the used DFT methodology, spin component-scaled (SCS) MP2 and $\operatorname{CCSD}(\mathrm{T})$ calculations were performed for cyclopropenes 1719 and 23-25 and for fluorinated o-quinones 26-28. Basically, these methods followed the same trends as the B97D calculations, and are only discussed where relevant; the complete SCS-MP2 and CCSD $(\mathrm{T})$ data are presented in the Supporting Information (Tables S2 and S6).)

Given the fast reaction measured for the strain-promoted reaction between $o$-quinones and 1-methyl-3-substituted cyclopropenes, we investigated the scope of this reaction using other strained cyclic alkenes, namely, cyclopropene (5), cyclobutene (6), cyclopentene (7), cyclohexene (8), and norbornene (9), to evaluate their potential use in this novel click reaction (Figure 2). In all cases, except for cyclohexene (8), the cycloaddition proceeds through a (near-)synchronous transition state with two identical C...C distances for the formed $\mathrm{C}-\mathrm{C}$ bonds, which decrease as the ring size of the cycloalkane increases (see Figure 2). The cyclopropene reaction has-as expected from the Hammond principlethe earliest transition state $\left(r(\mathrm{C} \cdots \mathrm{C})^{\mathrm{TS}}=2.35 \AA\right)$ and is the most exergonic reaction $(\Delta H=-32.7 \mathrm{kcal} / \mathrm{mol})$. On the other hand, the reaction with the cyclohexene, which is least loaded with steric strain, yields the latest TS and is less exothermic $\left(r(\mathrm{C} \cdots \mathrm{C})^{\mathrm{TS}}=2.43 \AA\right.$ and $-6.4 \mathrm{kcal} / \mathrm{mol}$, respectively $)$. According to the computed reaction profiles for the cycloaddition processes involving the set of cycloalkenes, the cyclopropene cycloaddition is clearly favored from both kinetic and thermodynamic points of view.

The energy barrier is also the lowest for cyclopropene (5.5 $\mathrm{kcal} / \mathrm{mol}$ ) in line with the activation by the ring strain in the reacting alkene. This observation is indeed reflected in the energy associated with the structural distortion of reagents to reach the transition state geometry. In the case of cyclopropene 5 and $o$-quinone, distortion energies of 7.3 and $8.7 \mathrm{kcal} / \mathrm{mol}$, respectively, were computed for these two compounds (Figure 2 ). For the rest of cycloalkenes 6-9, the distortion energies were almost 2 -fold higher, and in those TSs, the distortion energy of quinone 4 was also higher (increasing from $8.7 \mathrm{kcal} /$ mol for the reaction with 5 to $14.1 \mathrm{kcal} / \mathrm{mol}$ for the reaction with 6). To the degree that the reaction rate would be enthalpy-controlled, this would imply that the reaction rate of cyclopropenes will be orders of magnitude faster than that of any other cyclic cis-alkene.

Given the potential of this relatively novel reaction, we decided to investigate both counterparts, i.e., the cyclopropene and the quinone, in more detail. To this end, we studied a wide range of substituents on both the cyclopropene and the $o$ quinone.

Figure 3 shows the computed activation strain diagram (ASD) for the cycloaddition reactions between $o$-quinone and cyclopropene (black), cyclobutene (red), cyclopentene (blue), cyclohexene (green), and norbornene (magenta), from the respective initial reactant complexes to the corresponding transition states. Although all reactions exhibit rather similar diagrams, it becomes clear that the interaction between the deformed reactants is much stronger for the cycloaddition involving cyclohexene than for the analogous processes involving other cycloalkenes along the entire reaction coordinate. However, this stronger interaction is unable to compensate for the lower destabilizing strain energy computed for the reaction involving the cyclohexene and is therefore responsible for the higher barrier computed for the cyclohexene system.

Reactivity of Different Cyclopropene Derivatives. Electronic Effects on the Cyclopropene Ring. First, we 


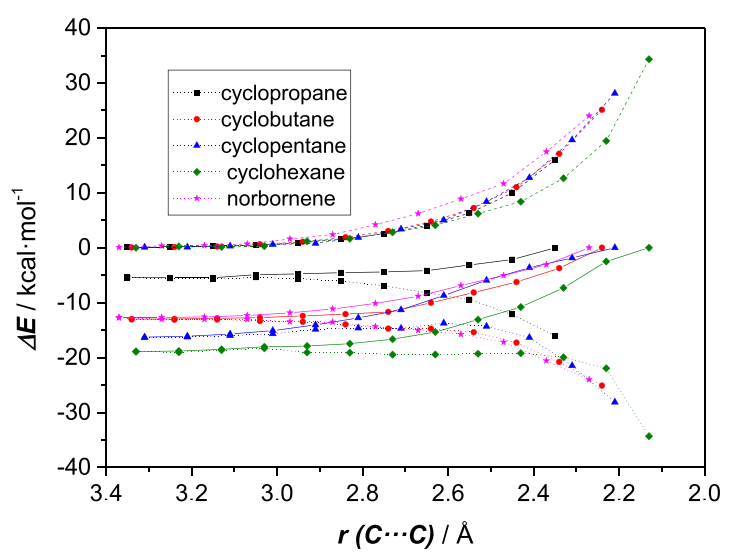

Figure 3. Comparative activation strain diagrams of the cycloaddition reaction involving $o$-quinone and cyclopropene (black), cyclobutene (red), cyclopentene (blue), cyclohexene (green), and norbornene (magenta). Activation energies (solid lines), distortion energies (dashed lines), and interaction energies (dotted lines) along the reaction coordinate projected onto the formed $\mathrm{C} \cdots \mathrm{C}$ bond distance. All data have been computed at the B97D/6-311+G(d,p) level of theory.

focused on one of the counterparts of the cycloaddition reaction: the cyclopropene. For that, a wide variety of cyclopropene derivatives were selected and evaluated in the cycloaddition reaction toward quinone 4 (see Figure S1 for an overview of all compounds). In the basic reaction of cyclopropene 5, the cycloaddition proceeds with an activation enthalpy of $4.5 \mathrm{kcal} / \mathrm{mol}$ through a synchronous transition state with a C...C distance of $2.35 \AA$. The presence of a methyl group at 1-position of the double bond in $\mathbf{1 0}$ increased the activation barrier $(5.3 \mathrm{kcal} / \mathrm{mol})$. This increase is accompanied by an earlier and nonsynchronous transition state with $\mathrm{C} \cdots \mathrm{C}$ distances of 2.11 and $2.71 \AA$. When analyzing these two TS geometries, a few trends can be observed. Starting with quinone 4, the $\mathrm{C} 2-\mathrm{C} 3$ distance (see Figure 4 for atom labeling) remains unchanged in TS-5 (1.47 A in the optimized geometry for quinone 4), but displays a slight variation in TS10 (1.45 and $1.49 \AA$ for $\mathrm{C} 2-\mathrm{C} 3$ and $\mathrm{C} 1-\mathrm{C} 6$, respectively). It is noteworthy that the lengthened bond is associated with the attack from $\mathrm{C} 2$ in cyclopropene 10. A look at the $\mathrm{C} 3-\mathrm{C} 4$ distance revealed a short lengthening from $1.37 \AA$ in quinone 4 to $1.40 \AA$ in TS-5 and TS-10. On the other hand, the C4-C5 distance was slightly shortened from $1.47 \AA$ in quinone 4 to $1.41 \AA$ in both TS structures. More significant variations were found when evaluating to which degree the quinone was bent, as a distortion of $24^{\circ}$ from the almost planar geometry ( $4^{\circ}$ of deviation for quinone 4) was calculated for the distorted quinone in TS-5. Given the unsymmetrical geometry of TS-10,
C3 and C6 were 27 and $18^{\circ}$ out of the planar geometry, respectively. When looking at the cyclopropene geometry, the C1-C2 double bond (1.31 $\AA$ ) was lengthened in the TS structures for 5 and 10, with distances of 1.36 and $1.38 \AA$, respectively. $\mathrm{C} 1-\mathrm{C} 2-\mathrm{H}$ changes from 150 to $142^{\circ}$ in TS-5, and from 150 to $144^{\circ}$ and $136^{\circ}$ for $\mathrm{C} 2-\mathrm{C} 1-\mathrm{C} 4$ and $\mathrm{C} 1-\mathrm{C} 2-\mathrm{H}$, respectively. Both $\mathrm{H}$ atoms at positions $\mathrm{C} 1$ and $\mathrm{C} 2$ in $\mathbf{5}$ are deviated $34^{\circ}$ from the original planar geometry; in contrast, for compound $10, \mathrm{H}$ and the $\mathrm{CH}_{3}$ group were bent 44 and $25^{\circ}$, respectively in agreement with the nonsynchronous transition state with the earlier formation of the $\mathrm{C}-\mathrm{C}$ at the $\mathrm{C} 2$ position.

A closer inspection at the distortion energies showed almost similar distortion energies for the $o$-quinone $(8.7 \mathrm{vs} 8.8 \mathrm{kcal} /$ mol for 5 and 10, respectively) but a high difference in the distortion energies for the cyclopropene (7.3 vs $9.7 \mathrm{kcal} / \mathrm{mol}$ for 5 and 10, respectively), which is reflected in the activation barrier. According to these B97D/6-311+G(d,p)-calculated barriers, 5 would react almost four times faster than 10 . Although cyclopropenes without substituents at the $\mathrm{C} 1$ position showed a higher reactivity with $o$-quinone, they also showed a limited stability due to rapid degradation. ${ }^{45}$ Consequently, we introduced a methyl group to the 1-position of the cyclopropene (both experimentally in ref 39 and in most other cyclopropenes under the current theoretical study) to improve their chemical stability while still conserving a high reactivity.

Next, we incorporated a higher degree of substitution on the cyclopropene via a range of 1,3-disubstituted cyclopropenes (see Figure 5) and evaluated - first by DFT - the effect of different groups at the $\mathrm{C} 3$ position; this 3-position in cyclopropenes is typically the position for introduction of diverse linker architectures with multiple applications in labeling and bioconjugation. ${ }^{46-49}$ Initially, we evaluated the effect of $\mathrm{CH}_{2}$-linked moieties at the $\mathrm{C} 3$ position for compounds bearing an ester (11), an amide (12), and an ether (13). When compared to the unsubstituted cyclopropene 10, higher activation enthalpies were found for compounds 11 and 12; on the contrary, for the $\mathrm{CH}_{2}$-linked ether derivative 13, this barrier decreased by $0.3 \mathrm{kcal} / \mathrm{mol}$. In all cases, the cycloaddition proceeded through a nonsynchronous transition state with $C \cdots C$ distances of 2.11 and $2.70 \AA$ (for 11 and 13) and 2.09 and $2.77 \AA$ (for 12), the latter having the highest activation energy. A deeper analysis of the distortion/ interaction energies revealed that the cyclopropene bearing the $\mathrm{CH}_{2}$-linked ether had lower distortion energies for cyclopropene and $o$-quinone when compared to $\mathbf{1 0}$, which is supported by a less distorted geometry in the TS structure. According to these calculations, compounds 10 and 13 would
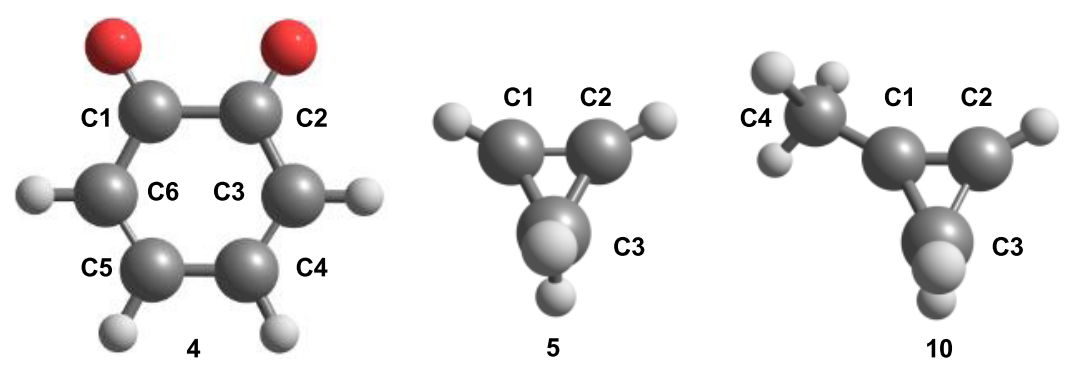

Figure 4. Labeling of atoms for compounds 4, 5, and 10. 


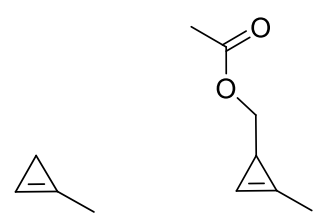

11

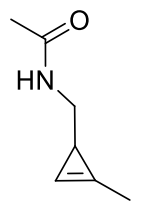

12

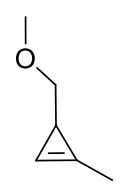

13
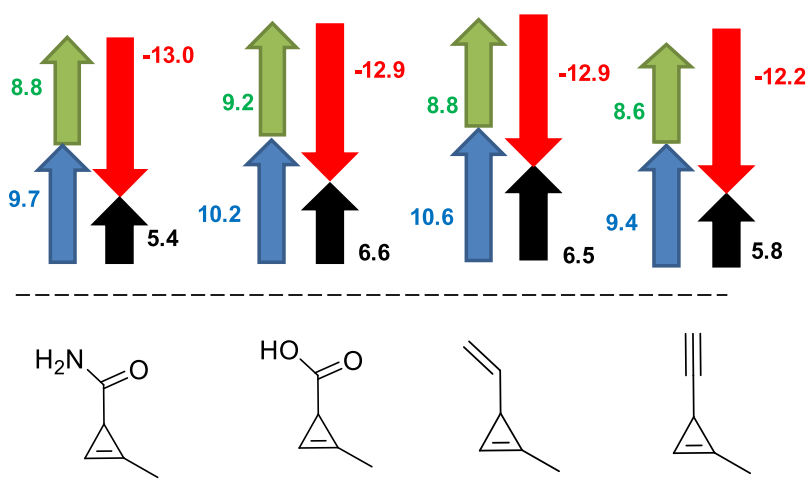

15
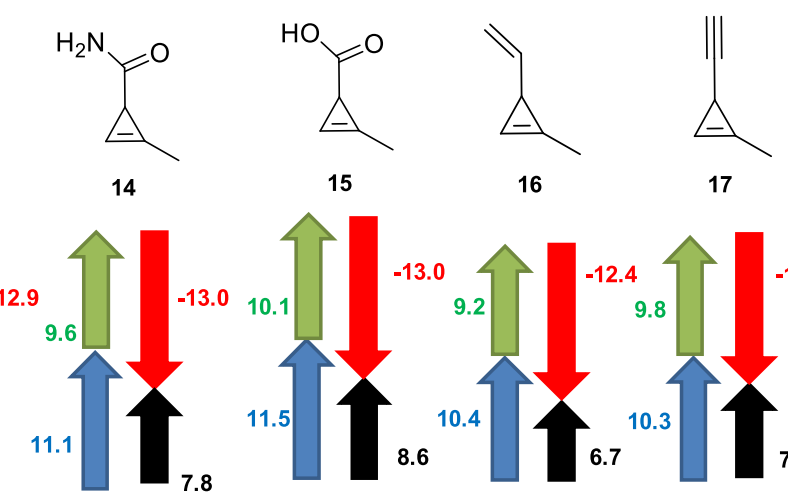

17

Figure 5. Distortion/interaction analysis for cycloaddition of 10-17 with 4 [B97D/6-311+G(d,p) data]. Cyclopropene distortion energy (blue arrow), $o$-quinone distortion energy (green arrow), activation enthalpy (black arrow), and interaction energy (red arrow). All energies are given in $\mathrm{kcal} / \mathrm{mol}$.

be expected to show a similar reactivity in the cycloaddition to $o$-quinone.

For compounds 14-17 (Figure 5), we found that moderately electron-withdrawing groups (EWG), such as carboxylic acid (14) or amide (15), increased the barrier by 2.6 and $1.7 \mathrm{kcal} / \mathrm{mol}$, respectively. However, as shown by Devaraj and co-workers, simple carboxyamide cyclopropene derivatives have a limited use in chemical biology due to the lack of stability, as they degraded quickly overnight at -20
${ }^{\circ} \mathrm{C} .{ }^{50}$ Second, the presence of an adjacent alkene $(16)$ or alkyne (17) increased the barrier by 0.8 and $1.7 \mathrm{kcal} / \mathrm{mol}$, respectively. These were also highly asynchronous, with C… C distances of 2.11 and $2.71 \AA$. Overall, the distortion/ interaction analysis showed that EWG groups diminish the reactivity slightly, which is largely due to increased distortion energies (see 14 and 15). The variation in the activation enthalpies caused by the effects of substituents at the 3position are similar to the trends in reactivity previously reported in inverse electron-demand Diels-Alder reactions with tetrazines and triazines. ${ }^{51}$

Steric Effects on the Cyclopropene. Next, the steric effects at the 1-position of the double bond were investigated (Figure 6). As mentioned above, the presence of a methyl group at the 1 -position of the internal double bond (13) increased the activation enthalpy barrier by $0.7 \mathrm{kcal} / \mathrm{mol}$ when compared to the homologous unsubstituted cyclopropene 18. For compound 18, the transition state showed a synchronous structure with a C ...C distance of $2.34 \AA$ and symmetrically distorted structures for the cyclopropene and $o$-quinone $\left(21^{\circ}\right.$ distorted from the planar geometry). Although the introduction of the methyl group has a negative effect on the kinetics of the reaction (vide supra), the starting 1-methylcyclopropenes are much more stable, facilitating experimental work. Consequently, we evaluated the steric effects on this position. Interestingly, the presence of an ethyl (19), propyl (20), or isopropyl (21) group did not have a significant effect on the activation barrier (Figure 5). A closer inspection of the TS structures reveals nonsynchronous transition states for

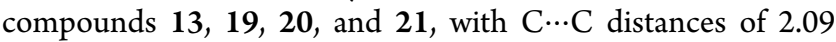
and $2.72 \AA$ for 13 and 19, respectively, being the shorter distance that corresponds to the unsubstituted 2-position. It is noteworthy that for cyclopropenes 20 and $\mathbf{2 1}$, the C...C distance for the new bond corresponding to the substituted 1of the TS, the $o$-quinone showed an unsymmetrically bended structure with deviations of 11 and $22^{\circ}$ from the planar geometry. On the other hand, the presence of methyl groups at both 1- and 2-positions of the double bond (22) resulted in a synchronous transition state with a C ...C distance of $2.31 \AA$ and symmetrically distorted structures for cyclopropene and $o$ quinone (with a distortion of $22^{\circ}$ from the planar geometry), which was reflected in an increase in the activation barrier by position increased to $2.82 \AA$. Given the asynchronous character
13

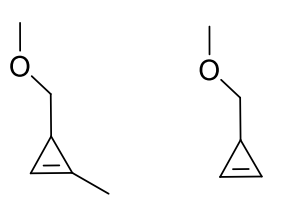

18

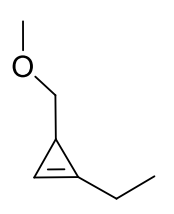

19

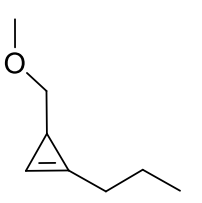

20

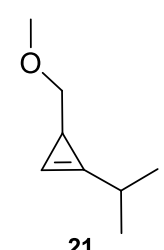

21

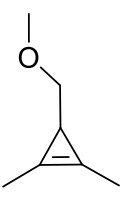

22
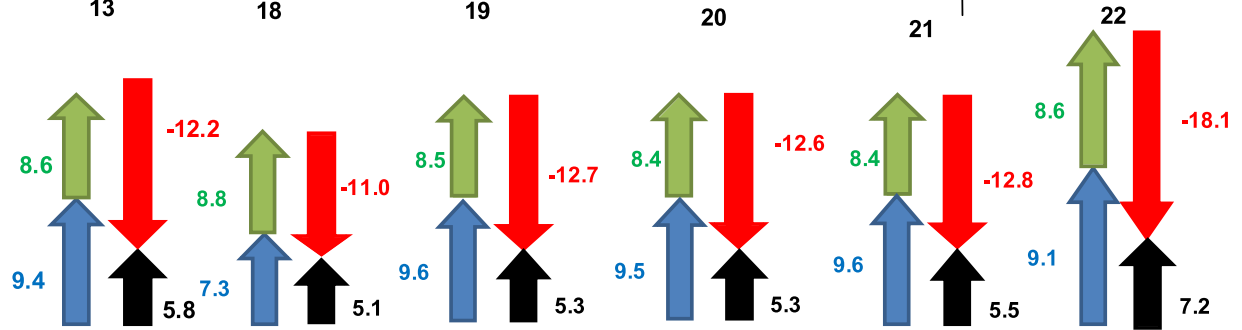

Figure 6. Distortion/interaction analysis for cycloaddition of 13 and 18-22 with 4 [B97D/6-311+G(d,p) data]. Cyclopropene distortion energy (blue arrow), quinone distortion energy (green arrow), activation enthalpy (black arrow), and interaction energy (red arrow). All energies are given in $\mathrm{kcal} / \mathrm{mol}$ 
$2.0 \mathrm{kcal} / \mathrm{mol}$ when compared to the unsubstituted cyclopropene 18. We analyzed the activation barriers for these reactions using the distortion/interaction model. In all cases, the $o$-quinone distortion energies were almost identical (between $8.4 \mathrm{kcal} / \mathrm{mol}$ for the propyl-substituted cyclopropenes 20 and 21 and $8.8 \mathrm{kcal} / \mathrm{mol}$ for the unsubstituted 18).

Finally, the effect of EWG groups at the 1-position of the double bond was also analyzed at the B97D/6-311+G(d,p) level of theory (Figure 7). Similar trends were observed at the

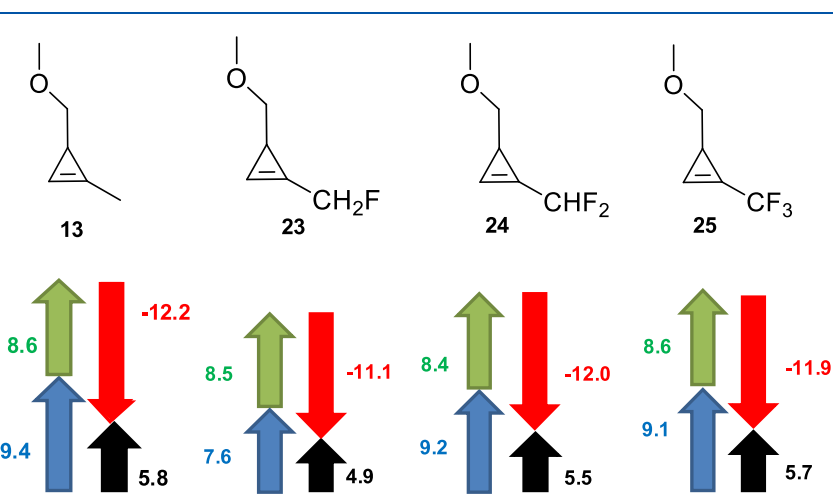

Figure 7. Distortion/interaction analysis for cycloaddition of 13, 23, 24, and 25 with $4[\mathrm{~B} 97 \mathrm{D} / 6-311+\mathrm{G}(\mathrm{d}, \mathrm{p})$ data]. Cyclopropene distortion energy (blue arrow), $o$-quinone distortion energy (green arrow), activation enthalpy (black arrow), and interaction energy (red arrow). All energies are given in $\mathrm{kcal} / \mathrm{mol}$.

SCS-MP2/def2-TZVP level of theory (see Tables S2 and S3 in the Supporting Information). For that purpose, the methyl group in this position (13) was replaced by a $\mathrm{CH}_{2} \mathrm{~F}(23)$, $\mathrm{CHF}_{2} \mathrm{~F}(24)$, or $\mathrm{CF}_{3}$ (25) group, which had a stabilizing effect on the TS as activation barriers were lowered by $0.4-1.1 \mathrm{kcal} /$ mol. In this regard, the incorporation of the 1-fluoromethyl group lowered the activation barrier to $4.3 \mathrm{kcal} / \mathrm{mol}$ compared to that of 1-methylcyclopropene (23). Incorporating more F atoms increased the enthalpic barrier up again to 4.9 and 5.0 $\mathrm{kcal} / \mathrm{mol}$ for the difluoromethyl (24) and trifluoromethyl (25) derivatives, respectively. For this set of fluorinated compounds, later and nonsynchronous transition states were observed with $\mathrm{C}-\mathrm{C}$ distances in the range of $2.2-2.5 \AA$. The fluorination effect is analogous to the azide cycloaddition with cyclooctyne and fluorinated compounds, namely, monofluorocyclooctyne (MOFO) and monofluorocyclooctyne (DIFO), as studied by Gold et al. ${ }^{52}$ As shown in Figure S2, monofluorination at the $\mathrm{CH}_{2}$ group adjacent to the triple bond increased the cyclopropene HOMO energy (reducing the energy gap) and might result in stabilization of the transition state through increased hyperconjugative interactions of the in-plane and out-of-plane $\pi$-bond of the cyclooctyne with the $\sigma^{*} \mathrm{C}-\mathrm{F}$ in the transition-state geometry, as described for the SPAAC reaction. However, in the case of cyclopropene derivatives, this effect is weaker relative to azides because cyclopropene contains only one $\pi$-bond. The incorporation of the second and third fluorine atoms provides only a small amount of stabilization because of the less than ideal overlap with the cyclopropene $\pi$ bond and the electron-withdrawing effects that lower the cyclopropene HOMO energy counter to the hyperconjugative stabilization, resulting in a lower reactivity compared to the methyl group. A good correlation between the activation enthalpy and the HOMO energy of the cyclopropene was observed $\left(\Delta H_{\text {methanol }}{ }^{\ddagger}=-2.71 E_{\mathrm{HOMO}}-20.93, R^{2}=0.9563\right.$; see Figure S2).

The distortion energies required for activation of the $o$ quinone are again very similar; however, increased fluorination increases the distortion energies of the cyclopropene derivatives. Here, the monofluoro $\mathrm{CH}_{2} \mathrm{~F}$ substituent seems to hold the sweet spot as its low cyclopropene distortion energy leads to an overall reduction in the activation enthalpy, also when compared to 13 , which makes this compound of interest for future experimental studies.

Reactivity of Different 0 -Quinones. Next, we focused on electronic variations in the $o$-quinone, using $\mathbf{1 3}$ as the constant cyclopropene (Scheme 2). To that end, we evaluated and compared the parent $o$-quinone 4 with fluorine substitution at the 3- and 6-positions (3,6-difluoro o-quinone, 26), at the 4and 5-positions (4,5-difluoro $o$-quinone, 27), and $o$-fluoranil (tetrafluoro-o-benzoquinone, 28), which has been previously studied by Lemal et al. in Diels-Alder reactions. ${ }^{53}$

Activation enthalpies were calculated at B97D/6-311+G(d,p), SCS-MP2/def2-TZVP, and $\operatorname{CCSD}(\mathrm{T}) / \mathrm{cc}-\mathrm{pVTZ} / /$ MP2/def2-TZVP levels of theory. TS optimization (at the different levels of calculation) showed that the cycloaddition proceeds through a nonsynchronous transition state with $\mathrm{C} \cdots \mathrm{C}$ distances of 2.04 and $2.15 \AA$, as shown in Figure 8. According to $B 97 D / 6-311+G(d, p)$ calculations, the incorporation of $F$ atoms at the 3- and 6-positions lowered the activation enthalpy slightly $(0.6 \mathrm{kcal} / \mathrm{mol})$ compared to 4 , whereas the presence of $\mathrm{F}$ atoms at the 4- and 5-positions increased the activation enthalpy $(1.7 \mathrm{kcal} / \mathrm{mol})$. In the case of the tetrafluorinated $o$ fluoranil, with the earliest TS, the activation enthalpy was the highest in the series, being $10.1 \mathrm{kcal} / \mathrm{mol}$. For SCS-MP2, similar activation barriers were computed, while for the singlepoint $\operatorname{CCSD}(\mathrm{T})$ calculations, the calculated barriers were 1.9$4.2 \mathrm{kcal} / \mathrm{mol}$ higher than those obtained using the B97D functional. If the $\operatorname{CCSD}(\mathrm{T})$ calculations are correct, then this would lead to a much larger dependence on the quinone structure than if B97D or SCS-MP2 data are correct; this clearly awaits $\operatorname{CCSD}(\mathrm{T})$ calculations in which the TS geometry

Scheme 2. Cyclopropene Cycloaddition with Different o-Quinones

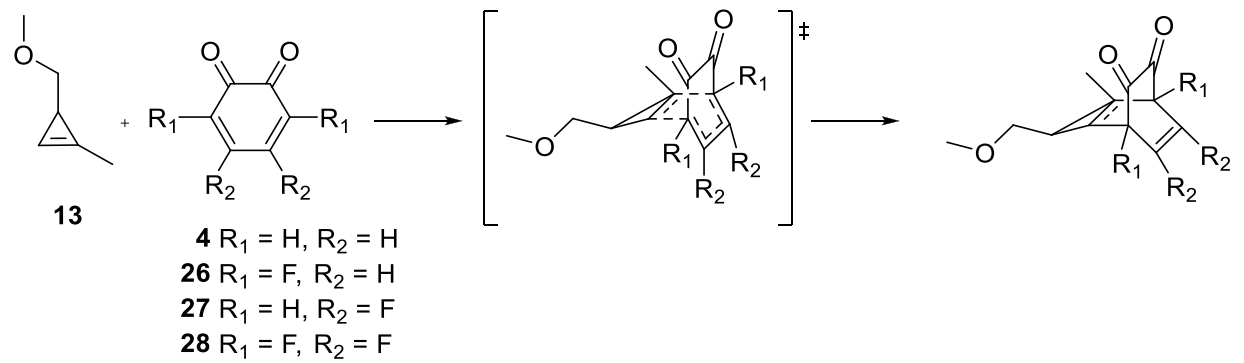




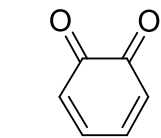

4<smiles>O=C1C(=O)C(F)=CC=C1F</smiles>

26<smiles>O=C1C=C(F)C(F)=CC1=O</smiles>

27<smiles>O=C1C(=O)C(F)=C(F)C(F)=C1F</smiles>

28
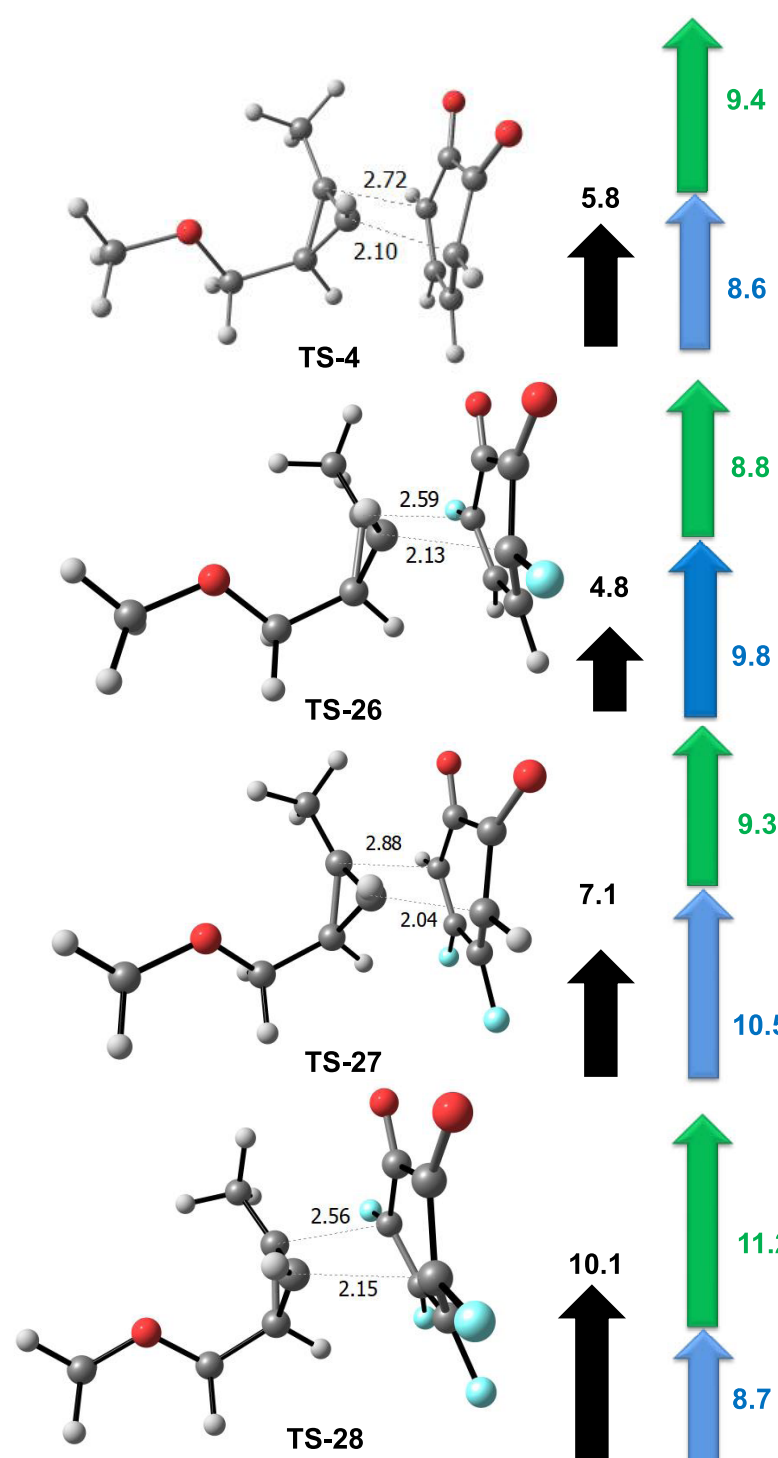

TS-27

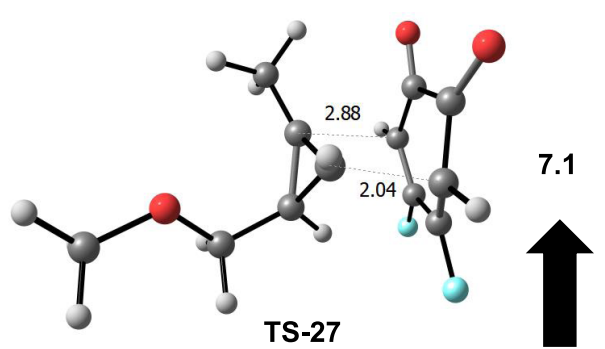

7.1

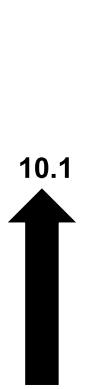

8.8

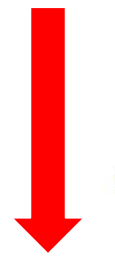

9.812 .4

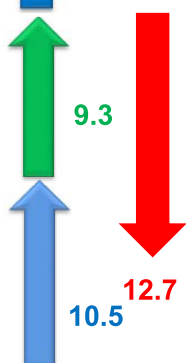

9.4

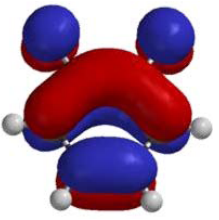

$E_{\text {LUMo }}=0.06 \mathrm{eV}$

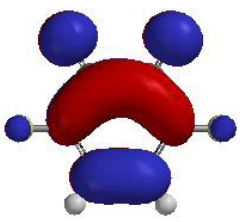

$E_{\text {LUMo }}=-0.42 \mathrm{eV}$

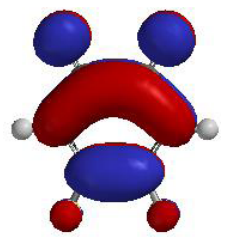

$E_{\text {LUмо }}=-0.18 \mathrm{eV}$

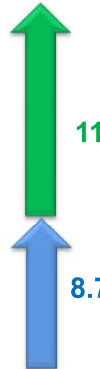

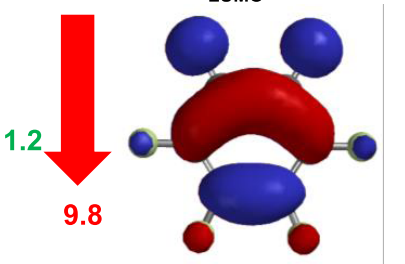

8.7
$E_{\text {LUMo }}=-0.66 \mathrm{eV}$

Figure 8. B97D/6-311+G(d,p)-optimized TS structures of cyclopropene 13 and fluorinated quinones 26 and 27. Activation enthalpies (in kcal/ $\mathrm{mol}$ ) computed at B97D/6-311+G(d,p). Cyclopropene distortion energy (blue arrow), o-quinone distortion energy (green arrow), activation enthalpy (black arrow), and interaction energy (red arrow). LUMO energies for ground-state quinones 4, 26, 27, and 28.

can also be optimized, as both the distortion and interaction parameters are highly geometry dependent.

A closer look at the activation strain diagrams of the cycloaddition reaction involving cyclopropene 17 and $o$ quinones 4, 26, 27, and 28 (Figure 9) reveals that all cycloadditions exhibit rather similar ASDs; it becomes clear that the interaction between the deformed reactants is much stronger for the cycloaddition involving 26 than for the analogous processes along the entire reaction coordinate.

The difference in reactivity can be rationalized through a closer inspection of the distortion energies. On one hand, for the highly reactive 3,6-fluorinated quinone 26 , the total distortion energy (B97D: $18.6 \mathrm{kcal} / \mathrm{mol}$; SCS-MP2: 14.9 $\mathrm{kcal} / \mathrm{mol}$ ) is slightly higher when compared to the nonfluorinated quinone (B97D: $18.0 \mathrm{kcal} / \mathrm{mol}$; SCS-MP2: 15.6 $\mathrm{kcal} / \mathrm{mol}$ ), which is associated with the lower distortion energy of the cyclopropene. On the other hand, for the slower 4,5fluorinated quinone 27, the distortion energy increased (B97D: $19.8 \mathrm{kcal} / \mathrm{mol}$; SCS-MP2: $19.2 \mathrm{kcal} / \mathrm{mol}$ ), which was obtained from a higher distortion energy of both the cyclopropene and the quinone. The electron-withdrawing groups at positions 3 and 6 in compound 26 lower the LUMO energy, decreasing the gap with the HOMO of cyclopropene, when compared to compound 27 with $\mathrm{F}$ atoms at positions 4 and $5(-0.42 \mathrm{vs}-0.18 \mathrm{eV})$. For the tetrafluorinated $o$-fluoranil, the total distortion energy was found to be higher (B97D: 21.7 $\mathrm{kcal} / \mathrm{mol}$ ) and the calculated LUMO energy was $-0.66 \mathrm{eV}$. Accordingly, we envision that 3,6-fluorinated o-quinone 26 would be a good candidate to be synthesized and studied experimentally in the cyclopropene cycloaddition due to its fast kinetics.

\section{CONCLUSIONS}

A quantum chemical study of the cycloaddition reaction between strained cyclic alkenes and ortho-quinones indicates that this novel click reaction proceeds concertedly with a rather synchronous formation of C...C bonds. A detailed distortioninteraction analysis reveals that for this type of reaction the distortion energy required to reach the transition state is typically of the same order as the overall interaction energy, 


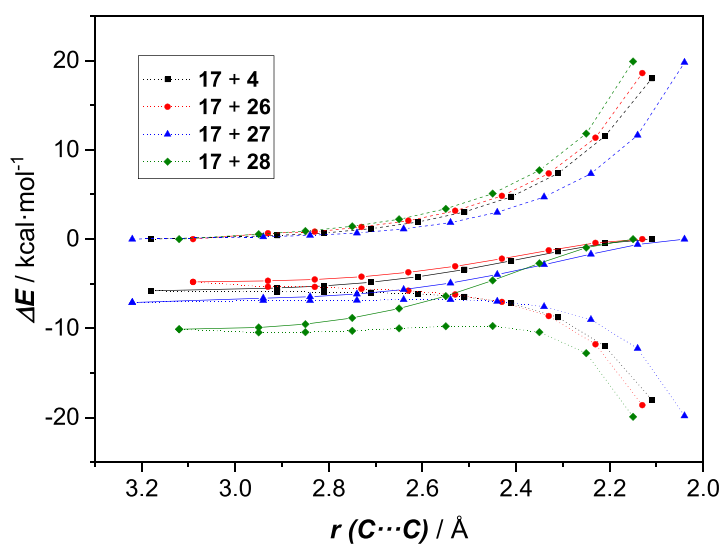

Figure 9. Comparative activation strain diagrams of the cycloaddition reaction involving cyclopropene 17 and $o$-quinones 4 (black), 26 (red), 27 (blue), and 28 (green). Activation energies (solid lines), distortion energies (dashed lines), and interaction energies (dotted lines) along the reaction coordinate projected onto the formed $\mathrm{C} \cdots \mathrm{C}$ bond distance. All data have been computed at the B97D/6$311+\mathrm{G}(\mathrm{d}, \mathrm{p})$ level of theory.

yielding low reaction enthalpic barriers throughout (typically 4-8 kcal/mol). Specifically, the use of well-placed fluorine atoms in both the cyclopropene and the ortho-quinone is evaluated to allow for an even further reduction in this barrier, showing the potential of this reaction within the tool box of click chemistries, especially given the small size of the cyclopropene compared to, e.g., activated trans-cyclooctenes and cyclooctynes.

\section{EXPERIMENTAL SECTION}

Computational Methods. All DFT geometry optimizations were performed with the dispersion-corrected B97D functional and 6$311+G(d, p)$ basis set as implemented within the Gaussian 16 series of programs. Solvent effects were included with the conductor-like polarizable continuum model (CPCM) to mimic methanol during both geometry optimizations and vibrational analysis. All of the energies and enthalpies presented for the reactant complex (RC), transition state (TS), and product (P) are given in Hartree. All of the energies have been corrected with zero-point energies (ZPE). Vibrational frequency calculations were made at the same level of theory used for optimization. All transition states were verified to have only one negative eigenvalue in the Hessian matrix, describing the motion along the reaction coordinate. Furthermore, the calculated activation enthalpies and reaction enthalpies are given for every reaction in $\mathrm{kcal} / \mathrm{mol}$. Every geometry on the reaction coordinates is separated into cycloalkene and quinone parts. Distortion energy is calculated as the energy difference between the energy of distorted cycloalkene and quinone parts and the energy of the fully optimized cycloalkene and quinone parts. The interaction energy is calculated as the energy difference between the energy of the geometry on the reaction coordinate and the energy of the relative distorted cycloalkene and quinone parts. When the distortion, interaction, and total energies are plotted, the formed $\mathrm{C} \cdots \mathrm{C}$ bond is used to represent the reaction coordinate.

$\mathrm{Ab}$ initio calculations for geometry optimization, frequency analysis, and thermodynamic calculations were based on SCS-MP2/ def2-TZVP and SCS-MP2/COSMO(PTE)/def2-TZVP calculations using the ORCA ${ }^{54}$ and Turbomole ${ }^{55}$ programs. Using the optimized geometries, basis set extrapolation to the complete basis set limit (CBS) was performed for activation energy barriers $\Delta E^{\ddagger}$, which for SCS-MP2 and DLPNO-CCSD $(T)$ were based on cc-pVTZ and ccpVQZ basis set calculations, respectively. These extrapolated $\Delta E_{\mathrm{CBS}} \neq$ values have been corrected for ZPE and thermal contributions and solvent effects as obtained using the SCS-MP2/def2-TZVP results. ${ }^{56}$
The resulting values are denoted $\Delta E_{\mathrm{CBS} \text {,solv }}{ }^{\neq}$for the respective method.

\section{ASSOCIATED CONTENT}

\section{Supporting Information}

The Supporting Information is available free of charge at https://pubs.acs.org/doi/10.1021/acs.joc.0c01674.

Computational details; compounds under current study; correlation between the activation enthalpy and HOMO of cyclopropene; reaction energetics and $\mathrm{C} \cdots \mathrm{C}$ bond lengths in TS; distortion/interaction analysis; NPA analysis using $\mathrm{B} 97 \mathrm{D} / 6-311+\mathrm{G}(\mathrm{d}, \mathrm{p})$ in the gas phase; Cartesian coordinates for optimized structures at B97D/ $6-311+G(d, p)$; and Cartesian coordinates for optimized structures at SCS-MP2/def2-TZVP/COSMO (PDF)

\section{AUTHOR INFORMATION}

\section{Corresponding Authors}

Hans Lischka - Department of Chemistry and Biochemistry, Texas Tech University, Lubbock, Texas 79409-1061, United States; School of Pharmaceutical Sciences and Technology, Tianjin University, Tianjin 300072, China; Institute for Theoretical Chemistry, University of Vienna, A-1090 Vienna, Austria; orcid.org/0000-0002-5656-3975; Email: hans.lischka@univie.ac.at

Han Zuilhof - Laboratory of Organic Chemistry, Wageningen University, 6708 WE Wageningen, The Netherlands; School of Pharmaceutical Sciences and Technology, Tianjin University, Tianjin 300072, China; Department of Chemical and Materials Engineering, Faculty of Engineering, King Abdulaziz University, 21589 Jeddah, Saudi Arabia; $\odot$ orcid.org/00000001-5773-8506; Email: han.zuilhof@wur.nl

\section{Authors}

Jorge Escorihuela - Departament de Química Orgànica, Universitat de Vatencia, 46100 Burjassot, Valencia, Spain; (1) orcid.org/0000-0001-6756-0991

Wilhelmus J. E. Looijen - Laboratory of Organic Chemistry, Wageningen University, 6708 WE Wageningen, The Netherlands; 10 orcid.org/0000-0001-5944-822X

Xiao Wang - School of Pharmaceutical Sciences and Technology, Tianjin University, Tianjin 300072, China

Adelia J. A. Aquino - Department of Mechanical Engineering, Texas Tech University, Lubbock, Texas 79409, United States; School of Pharmaceutical Sciences and Technology, Tianjin University, Tianjin 300072, China; Institute for Soil Research, University of Natural Resources and Life Sciences, A-1190 Vienna, Austria; 이이이.org/0000-0003-4891-6512

Complete contact information is available at: https://pubs.acs.org/10.1021/acs.joc.0c01674

\section{Notes}

The authors declare no competing financial interest.

\section{ACKNOWLEDGMENTS}

The authors are grateful for generous support by the School of Pharmaceutical Science and Technology, Tianjin University, China, including computer time on the SPST computer cluster Arran and for computer time at the Vienna Scientific Cluster (VSC). 


\section{REFERENCES}

(1) Kolb, H. C.; Finn, M. G.; Sharpless, K. B. Click Chemistry: Diverse Chemical Function from a Few Good Reactions. Angew. Chem., Int. Ed. 2001, 40, 2004-2021.

(2) Tiwari, V. K.; Mishra, B. B.; Mishra, K. B.; Mishra, N.; Singh, A. S.; Chen, X. Cu-Catalyzed Click Reaction in Carbohydrate Chemistry. Chem. Rev. 2016, 116, 3086-3240.

(3) He, X.-P.; Zeng, Y.-L.; Zang, Y.; Li, J.; Field, R. A.; Chen, G.-R. Carbohydrate CuAAC click chemistry for therapy and diagnosis. Carbohydr. Res. 2016, 429, 1-22.

(4) Döhler, D.; Michael, P.; Binder, W. H. CuAAC-Based Click Chemistry in Self-Healing Polymers. Acc. Chem. Res. 2017, 50, 26102620.

(5) Arslan, M.; Acik, G.; Tasdelen, M. A. The emerging applications of click chemistry reactions in the modification of industrial polymers. Polym. Chem. 2019, 10, 3806-3821.

(6) Meyer, J.-P.; Adumeau, P.; Lewis, J. S.; Zeglis, B. M. Click Chemistry and Radiochemistry: The First 10 Years. Bioconjugate Chem. 2016, 27, 2791-2807.

(7) Urkow, J.; Bergmana, C.; Wuest, F. Sulfo-click chemistry with ${ }^{18}$ F-labeled thio acids. Chem. Commun. 2019, 55, 1310-1313.

(8) Haldón, E.; Nicasio, M. C.; Pérez, P. J. Copper-catalysed azidealkyne cycloadditions (CuAAC): an update. Org. Biomol. Chem. 2015, $13,9528-9550$.

(9) Liang, L.; Astruc, D. The copper(I)-catalyzed alkyne-azide cycloaddition (CuAAC) "click" reaction and its applications. An overview. Coord. Chem. Rev. 2011, 255, 2933-2945.

(10) Clark, P. M.; Dweck, J. F.; Mason, D. E.; Hart, C. R.; Buck, S. B.; Peters, E. C.; Agnew, B. J.; Hsieh-Wilson, L. C. Direct In-Gel Fluorescence Detection and Cellular Imaging of O-GlcNAc-Modified Proteins. J. Am. Chem. Soc. 2008, 130, 11576-11577.

(11) Sawa, M.; Hsu, T.-L.; Itoh, T.; Sugiyama, M.; Hanson, S. R.; Vogt, P. K.; Wong, C.-H. Glycoproteomic probes for fluorescent imaging of fucosylated glycans in vivo. Proc. Natl. Acad. Sci. U.S.A. 2006, 103, 12371-12376.

(12) Tajmir-Riahi, H. A.; Langlais, M.; Savoie, R. A laser Raman spectroscopic study of the interaction of calf-thymus DNA with $\mathrm{Cu}(\mathrm{II})$ and $\mathrm{Pb}(\mathrm{II})$ ions: metal ion binding and DNA conformational changes. Nucleic Acids Res. 1988, 16, 751-762.

(13) Escorihuela, J.; Marcelis, A. T. M.; Zuilhof, H. Metal-Free Click Chemistry Reactions on Surfaces. Adv. Mater. Interfaces 2015, 2, No. 1500135

(14) McNitt, C. D.; Cheng, H.; Ullrich, S.; Popik, V. V.; Bjerknes, M. Multiphoton Activation of Photo-Strain-Promoted Azide Alkyne Cycloaddition "Click" Reagents Enables in Situ Labeling with Submicrometer Resolution. J. Am. Chem. Soc. 2017, 139, 1402914032 .

(15) Escorihuela, J.; Bañus, M. J.; Puchades, R.; Maquieira, A. Sitespecific immobilization of DNA on silicon surfaces by using the thiol-yne reaction. J. Mater. Chem. B 2014, 2, 8510-8517.

(16) Hoyle, C. E.; Lowe, A. B.; Bowman, C. N. Thiol-click chemistry: a multifaceted toolbox for small molecule and polymer synthesis. Chem. Soc. Rev. 2010, 39, 1355-1387.

(17) Collins, J.; Xiao, Z.; Müllner, M.; Connal, L. A. The emergence of oxime click chemistry and its utility in polymer science. Polym. Chem. 2016, 7, 3812-3826.

(18) Nair, D. P.; Podgórski, M.; Chatani, S.; Gong, T.; Xi, W.; Fenoli, C. R.; Bowman, C. N. The Thiol-Michael Addition Click Reaction: A Powerful and Widely Used Tool in Materials Chemistry. Chem. Mater. 2014, 26, 724-744.

(19) Dong, J.; Krasnova, L.; Finn, M. G.; Sharpless, K. B. Sulfur(VI) Fluoride Exchange (SuFEx): Another Good Reaction for Click Chemistry. Angew. Chem., Int. Ed. 2014, 53, 9430-9448.

(20) Gahtory, D.; Sen, R.; Pujari, S.; Li, S.; Zheng, Q.; Moses, J. E.; Sharpless, K. B.; Zuilhof, H. Quantitative and Orthogonal Formation and Reactivity of SuFEx Platforms. Chem. - Eur. J. 2018, 24, 1055010556.

(21) Liang, D.; Streefkerk, D. E.; Jordaan, D.; Wagemakers, J.; Baggerman, J.; Zuilhof, H. Silicon-Free SuFEx Reactions of
Sulfonimidoyl Fluorides: Scope, Enantioselectivity, and Mechanism. Angew. Chem., Int. Ed. 2020, 59, 7494-7500.

(22) Barrow, A. S.; Smedley, C. J.; Zheng, Q.; Li, S.; Dong, J.; Moses, J. E. The growing applications of SuFEx click chemistry. Chem. Soc. Rev. 2019, 48, 4731-4758.

(23) Dommerholt, J.; Rutjes, F. P. J. T.; van Delft, F. L. StrainPromoted 1,3-Dipolar Cycloaddition of Cycloalkynes and Organic Azides. Top. Curr. Chem. 2016, 374, No. 16.

(24) Harris, T.; Alabugin, I. V. Strain and stereoelectronics in cycloalkyne click chemistry. Mendeleev Commun. 2019, 29, 237-248.

(25) Sen, R.; Escorihuela, J.; Smulders, M. M. J.; Zuilhof, H. Use of Ambient Ionization High-Resolution Mass Spectrometry for the Kinetic Analysis of Organic Surface Reactions. Langmuir 2016, 32, 3412-3419.

(26) Blackman, M. L.; Royzen, M.; Fox, J. M. Tetrazine ligation: fast bioconjugation based on inverse-electron-demand Diels-Alder reactivity. J. Am. Chem. Soc. 2008, 130, 13518-13519.

(27) Sen, R.; Gahtory, D.; Escorihuela, J.; Firet, J.; Pujari, S.; P. Zuilhof, H. Approach Matters: The Kinetics of Interfacial InverseElectron Demand Diels-Alder Reactions. Chem. - Eur. J. 2017, 23, 13015-13022.

(28) Oliveira, B. L.; Guo, Z.; Bernardes, G. J. L. Inverse electron demand Diels-Alder reactions in chemical biology. Chem. Soc. Rev. 2017, 46, 4895-4950.

(29) Png, Z. M.; Zeng, H.; Ye, Q.; Xu, J. Inverse-Electron-Demand Diels-Alder Reactions: Principles and Applications. Chem. Asian J. 2017, 12, 2142-2159.

(30) Zanda, M.; Bucci, R.; Sloan, N. L.; Topping, L. Highly Strained Unsaturated Carbocycles. Eur. J. Org. Chem. 2020, 2020, 5278-5291.

(31) MacKenzie, D. A.; Sherratt, A. R.; Chigrinova, M.; Cheung, L. L. W.; Pezacki, J. P. Strain-promoted cycloadditions involving nitrones and alkynes-rapid tunable reactions for bioorthogonal labeling. Curr. Opin. Chem. Biol. 2014, 21, 81-88.

(32) Ning, X.; Temming, R. P.; Dommerholt, J.; Guo, J.; Ania, D. B.; Debets, M. F.; Wolfert, M. A.; Boons, G.-J.; van Delft, F. L. Protein modification by strain-promoted alkyne-nitrone cycloaddition. Angew. Chem., Int. Ed. 2010, 49, 3065-3068.

(33) Jonker, A. M.; Borrmann, A.; van Eck, E. R. H.; van Delft, F. L.; Lowik, D. W. P. M.; van Hest, J. C. M. A Fast and Activatable CrossLinking Strategy for Hydrogel Formation. Adv. Mater. 2015, 27, $1235-1240$.

(34) Borrmann, A.; Fatunsin, O.; Dommerholt, J.; Jonker, A. M.; Lowik, D. W. P. M.; van Hest, J. C. M.; van Delft, F. L. StrainPromoted Oxidation-Controlled Cyclooctyne-1,2-Quinone Cycloaddition (SPOCQ) for Fast and Activatable Protein Conjugation. Bioconjugate Chem. 2015, 26, 257-261.

(35) Sen, R.; Escorihuela, J.; van Delft, F.; Zuilhof, H. Rapid and Complete Surface Modification with Strain-Promoted OxidationControlled Cyclooctyne-1,2-Quinone Cycloaddition (SPOCQ). Angew. Chem., Int. Ed. 2017, 56, 3299-3303.

(36) Escorihuela, J.; Das, A.; Looijen, W. J. E.; van Delft, F. L.; Aquino, A. J. A.; Lischka, H.; Zuilhof, H. Kinetics of the StrainPromoted Oxidation-Controlled Cycloalkyne-1,2-quinone Cycloaddition: Experimental and Theoretical Studies. J. Org. Chem. 2018, 83, 244-252.

(37) Bruins, J. J.; Albada, B.; van Delft, F. ortho-Quinones and Analogues Thereof: Highly Reactive Intermediates for Fast and Selective Biofunctionalization. Chem. - Eur. J. 2018, 24, 4749-4756.

(38) Ravasco, J. M. J. M.; Monteiro, C. M.; Trindade, A. F. Cyclopropenes: a new tool for the study of biological systems. Org. Chem. Front. 2017, 4, 1167-1198.

(39) Gahtory, D.; Sen, R.; Kuzmyn, A. R.; Escorihuela, J.; Zuilhof, H. Strain-Promoted Cycloaddition of Cyclopropenes with $o$-Quinones: A Rapid Click Reaction. Angew. Chem., Int. Ed. 2018, 57, 10118-10122.

(40) Stephanopoulos, N.; Francis, M. B. Choosing an effective protein bioconjugation strategy. Nat. Chem. Biol. 2011, 7, 876-884.

(41) Fernández-Ramos, A.; Miller, J. A.; Klippenstein, S. J.; Truhlar, D. G. Modeling the Kinetics of Bimolecular Reactions. Chem. Rev. 2006, 106, 4518-4584. 
(42) Cheng, G.-J.; Zhang, X.; Chung, L. W.; Xu, L.; Wu, Y.-D. Computational Organic Chemistry: Bridging Theory and Experiment in Establishing the Mechanisms of Chemical Reactions. J. Am. Chem. Soc. 2015, 137, 1706-1725.

(43) Bickelhaupt, F. M.; Houk, K. N. Analyzing Reaction Rates with the Distortion/Interaction-Activation Strain Model. Angew. Chem., Int. Ed. 2017, 56, 10070-10086.

(44) Hamlin, T. A.; Levandowski, B. J.; Narsaria, A. K.; Houk, K. N.; Bickelhaupt, F. M. Structural Distortion of Cycloalkynes Influences Cycloaddition Rates both by Strain and Interaction Energies. Chem. Eur. J. 2019, 25, 6342-6348.

(45) Yang, J.; Liang, Y.; Šečkute, J.; Houk, K. N.; Devaraj, N. K. Synthesis and reactivity comparisons of 1-methyl-3-substituted cyclopropene mini-tags for tetrazine bioorthogonal reactions. Chem. - Eur. J. 2014, 20, 3365-3375.

(46) Patterson, D. M.; Nazarova, L. A.; Xie, B.; Kamber, D. N.; Prescher, J. A. Functionalized Cyclopropenes As Bioorthogonal Chemical Reporters. J. Am. Chem. Soc. 2012, 134, 18638-18643.

(47) Oller-Salvia, B.; Kym, G.; Chin, J. W. Rapid and Efficient Generation of Stable Antibody-Drug Conjugates via an Encoded Cyclopropene and an Inverse-Electron-Demand Diels-Alder Reaction. Angew. Chem., Int. Ed. 2018, 57, 2831-2834.

(48) Reisacher, U.; Ploschik, D.; Rönicke, F.; Cserép, G. B.; Kele, P.; Wagenknecht, H.-A. Copper-free dual labeling of DNA by triazines and cyclopropenes as minimal orthogonal and bioorthogonal functions. Chem. Sci. 2019, 10, 4032-4037.

(49) Wørmer, G. J.; Hansen, B. K.; Palmfeldt, J.; Poulsen, T. B. A Cyclopropene Electrophile that Targets Glutathione S-Transferase Omega-1 in Cells. Angew. Chem., Int. Ed. 2019, 58, 11918-11922.

(50) Yang, J.; Seckute, J.; Cole, C. M.; Devaraj, N. K. Live-cell imaging of cyclopropene tags with fluorogenic tetrazine cycloadditions. Angew. Chem., Int. Ed. 2012, 51, 7476-7479.

(51) Zhang, J.; Shukla, V.; Boger, D. L. Inverse Electron Demand Diels-Alder Reactions of Heterocyclic Azadienes, 1-Aza-1,3-Butadienes, Cyclopropenone Ketals, and Related Systems. A Retrospective. J. Org. Chem. 2019, 84, 9397-9445.

(52) Gold, B.; Shevchenko, N. E.; Bonus, N.; Dudley, G. B.; Alabugin, I. V. Selective Transition State Stabilization via Hyperconjugative and Conjugative Assistance: Stereoelectronic Concept for Copper-Free Click Chemistry. J. Org. Chem. 2012, 77, 75-89.

(53) Lemal, D. M.; Sang, D.; Ramanathan, S. o-Fluoranil: Stereochemistry and Mechanism of Its Diels-Alder Reactions. J. Org. Chem. 2009, 74, 7804-7811.

(54) Neese, F. The ORCA program system. Wiley Interdiscip. Rev.: Comput. Mol. Sci. 2012, 2, 73-78.

(55) TURBOMOLE, version 6.2; University of Karlsruhe and Forschungszentrum Karlsruhe GmbH, 1989-2007, 2010.

(56) Rappoport, D.; Furche, F. Property-optimized Gaussian basis sets for molecular response calculations. J. Chem. Phys. 2010, 133, No. 134105. 